\title{
Algoritmo de desactivación de estaciones base para reducir el consumo de energía en redes celulares
}

\author{
Zury Jeheili Santiago Manzano ${ }^{1}$, Anabel Martínez $\operatorname{Vargas}^{1}$, Ángel G. Andrade ${ }^{2}$ \\ ${ }^{1}$ Universidad Politécnica de Pachuca, Zempoala, Hidalgo, México \\ ${ }^{2}$ Universidad Autónoma de Baja California, Facultad de Ingeniería, Mexicali, \\ Baja California, México
}

zury_santiago@micorreo.upp.edu.mx, anabel.martinez@upp.edu.mx, aandrade@uabc.edu.mx

Resumen. Los teléfonos inteligentes se han convertido en dispositivos indispensables para las actividades cotidianas que realiza el ser humano. Su proliferación ha incrementado la infraestructura celular en un esfuerzo de los operadores móviles por satisfacer la demanda. Dado que la planificación de las redes celulares se realiza considerando la demanda en horas pico, se despliegan un gran número de estaciones base, manteniendo el mismo número de estaciones base aun cuando la intensidad de tráfico se reduzca. Esta estrategia ha traído consigo que se eleven los niveles de energía en las redes celulares, afectando no solo a sus gastos operativos sino también contribuyendo con las emisiones de carbono a la atmósfera. En el presente trabajo se muestra un algoritmo de desactivación de estaciones base para ahorrar energía en una red celular considerando la reasignación de usuarios móviles. Para encontrar el conjunto de estaciones base que deberán desactivarse y seguir ofreciendo los servicios demandados, se utiliza un algoritmo genético con modelo de población estacionario.

Palabras clave: algoritmo genético, redes verdes, LTE, ahorro de energía.

\section{Base Station Switching On/Off Algorithm to Reduce Energy Consumption in Cellular Networks}

\begin{abstract}
Smartphones have become essential devices for the daily activities that the human being performs. Its proliferation has increased the cellular infrastructure in an effort of the mobile operators to satisfy the demand. Due to the cellular network planning is performed considering the peak hour, a large number of base stations are deployed, maintaining the same number of base stations even in low traffic states. This strategy led to high levels of energy in cellular networks, affecting their operating expenditure and contributing to $\mathrm{CO}_{2}$ emissions. The present work shows a base station switching on/off algorithm to save energy in a cellular network considering the reassignment of mobile users.
\end{abstract}


In order to find out the base stations to switch off, a genetic algorithm with steady-state population model is used.

Keywords: genetic algorithm, green networks, LTE, energy saving.

\section{Introducción}

Los teléfonos inteligentes se han convertido en dispositivos indispensables para las actividades cotidianas que realiza el ser humano. Se estima que para el año 2020, el $72 \%$ de la población mundial contará con un dispositivo móvil [1]. La planificación de las redes celulares se realiza considerando el peor escenario, es decir, la demanda en horas pico [2]. Esto implica que se despliegue un gran número de estaciones base para atender estados de tráfico altos, sin embargo, este mismo número de estaciones base se mantiene aun cuando se reduzca la intensidad del tráfico. Lo anterior constituye un crecimiento en los niveles de consumo de energía de los operadores, elevando su gasto operativo. Así mismo, el alto consumo de energía resulta en que las redes celulares contribuyan con el $0.2 \%$ de emisiones de carbono del total del $2 \%$ que la industria de las Tecnologías de la Información y Comunicaciones (TIC) emiten a la atmósfera [3]. Ante estas problemáticas, una solución propuesta son las llamadas redes verdes que como su nombre lo sugiere son redes celulares amigables con el medio ambiente. Basadas en el hecho de que la estación base (BS) es el elemento que más energía consume (aproximadamente el 57\% de la energía total consumida por una red celular), los esfuerzos se han concentrado en técnicas para mejorar su eficiencia energética manteniendo los requerimientos de Calidad de Servicio (QoS) de los usuarios móviles [3]. Estas técnicas se centran en dos aspectos: 1) soluciones de hardware para mejorar el consumo de energía de los componentes de la BS (amplificadores de potencia, procesadores de señales digitales, sistemas de enfriamiento, cables alimentadores); y 2) administración inteligente de los elementos de la red basada en las variaciones de carga de tráfico. La técnica de apagado y encendido de las BS pertenecen a esta última categoría, logrando reducir entre $12-40 \%$ del consumo de energía en una red celular [4].

En la literatura se han reportado diversos trabajos que proponen enfoques para activar y desactivar las BS. Por ejemplo, en [2] se propone una planeación de redes verdes LTE (Long-Term Evolution) para minimizar las BS activas y definir los patrones de apagado basándose en el cambio de las condiciones de tráfico en la red. Los aspectos que considera son la cobertura de las BS, el número máximo de usuarios móviles a los que una BS puede servir y la probabilidad de interrupción. Para redes pequeñas y medianas, el problema es transformado a uno de programación lineal entera y se resuelve con un algoritmo de ramificación y poda. Para redes de mayor tamaño se propone una solución heurística. Es conocido que la eficiencia de los algoritmos de ramificación y poda depende de los algoritmos usados para cada uno de estos procesos, para evitar una repetida ramificación, sin poda.

En [5], bajo la premisa de que apagar las BS en un orden especifico y no precisamente comenzando por las BS de menor carga permite que un menor número de BS permanezcan activas, los autores proponen un enfoque para minimizar el número 
de BS activas. Presenta cuatro restricciones para la desactivación, que son: ancho de banda de la BS, BS desactivadas, cobertura y probabilidad de interrupción. Se aplica un algoritmo genético y se realiza la reasignación de usuarios móviles, aunque no está explícitamente indicado como se lleva a cabo.

De igual forma, en [6] se aplica un algoritmo de sociedad de arañas para resolver el problema de apagado y encendido de las BS en el que se minimiza el número de las BS activas. Para apagar una BS, el algoritmo penaliza de acuerdo a las BS vecinas disponibles. Si las BS vecinas a las que se desea apagar tienen recursos disponibles para recibir a más usuarios móviles el valor de aptitud de esta BS será menor, en caso contrario, será superior. Al disminuir la aptitud la solución será considerada más adecuada para el problema. El algoritmo de sociedad de arañas se modificó para resolver el problema en espacio discreto, ya que originalmente está diseñado para resolver problemas de optimización en espacio continuo. En [7] se argumenta que transformar un algoritmo de optimización que ha sido diseñado para resolver problemas en espacio continuo, a uno en espacio discreto, afecta su desempeño.

Así también en [8] se presenta un esquema que considera la operación dinámica de las BS, así como el problema de asociación de usuarios. En este trabajo se modela el problema de operación de BS dinámicas (apagar o encender una BS considerando el tráfico de carga) como una función de aptitud que minimiza el consumo de energía y el nivel de flujo de datos. Se derivan dos problemas: 1) La asignación de los usuarios móviles, para lo que se debe definir una política que garantiza que los usuarios móviles se asocien con las BS de una manera energéticamente eficiente, teniendo en cuenta el equilibrio de carga; y, 2) El apagado de las BS que se resuelve mediante un algoritmo voraz. Sin embargo, un algoritmo voraz analiza todas las posibles soluciones al problema lo que es muy costoso computacionalmente.

Un algoritmo que decide el apagado y encendido de las BS en una red heterogénea (red celular y red inalámbrica de área local) se propone en [9]. Se define una función de costo que minimiza el consumo de energía permitiendo un mayor número de accesos a la red. Aplican dos algoritmos codiciosos: el primero basado en la función de costo y el segundo basado en la densidad de puntos de acceso dentro de la cobertura de cada BS (apaga las BS con mayor número de usuarios móviles asociados). No obstante, el uso de algoritmos codiciosos tiene un costo computacional muy alto [10].

En [3] se propone redimensionar una red verde LTE, determinando el número mínimo de BS activas dada una determinada carga de tráfico con restricciones de QoS. La reducción de energía se representa por el número de las BS activas. Se aplica un algoritmo genético con elitismo para resolver el problema. El elitismo en algunos problemas lleva a una convergencia prematura, así como a un desempeño pobre en problemas que tienen una alta dimensionalidad.

Por último, el trabajo presentado en [11] reduce el consumo de energía en una red celular mediante un algoritmo de apagado de BS estableciendo que las BS se desactiven una a la vez, puesto que así se afecta mínimamente la carga de las demás BS; cada que se apague una BS se evaluará el incremento de carga en las BS vecinas. Para ello, toma en cuenta el tipo de región (urbana, metropolitana, etc.), ubicación de las BS y su cobertura. Se propone un algoritmo secuencial llamado SWES para reducir el tráfico 
de señalización evaluando el impacto que tendrá en la red el apagado de determinada BS.

En este trabajo se propone un algoritmo para reducir el consumo de energía en una red verde LTE mediante el apagado y encendido de las BS. La mínima cantidad de BS activas estarán en función de la carga de tráfico actual (número de usuarios móviles activos). Es un problema de optimización NP-duro [2] cuyo objetivo es minimizar el número de estaciones bases activas para reducir el consumo de energía. La motivación para usar este enfoque viene de las fluctuaciones de tráfico que se dan a lo largo de un día, donde cargas bajas de tráfico no necesitan mantener todas las estaciones bases activas. El algoritmo propuesto considera la reasignación de usuarios una vez que una estación base es desactivada.

Los recursos de las BS están disponibles en un instante de tiempo, entonces el algoritmo evalúa el escenario de red en ese momento, desactivando algunas BS. Al desactivar una BS, busca reasignar sus usuarios móviles a las BS vecinas. Enseguida, analiza si con la selección de BS activas y reasignación es suficiente para atender al $99 \%$ de los usuarios móviles presentes en ese instante de tiempo. Con ello se garantizará que con las BS que aún permanecen activas se seguirán ofreciendo los servicios a los usuarios móviles.

Para la búsqueda de una solución se utiliza un algoritmo genético (AG) con modelo de población estacionario [12]. Un AG permite tratar instancias del problema de gran tamaño, encontrando soluciones satisfactorias en un tiempo razonable [10]. Durante el proceso de búsqueda de una solución, un AG imita el proceso natural de supervivencia del más fuerte. Evoluciona una población de soluciones aplicando iterativamente operaciones de selección, cruzamiento y mutación. Una vez que el proceso iterativo concluye, el individuo más apto es la solución del problema. A diferencia de un AG con modelo de población generacional donde la población de individuos es reemplazada por los hijos en cada generación, un AG con modelo de población estacionario remplaza solo una parte de la población de individuos. Esto reduce el tiempo de búsqueda de una solución.

Aunque algunas de las propuestas que se describieron anteriormente tratan indirectamente la complejidad del problema transformándolo en otro cuya solución óptima es menos difícil de encontrar, la presente propuesta lo trata directamente usando un AG. Por otro lado, los AG están diseñados para trabajar en espacios discretos, por lo cual tampoco es necesaria alguna transformación en su proceso de búsqueda de una solución. La implementación del AG con modelo de población estacionario, reduce el tiempo de búsqueda de una solución ya que no procesa a toda la población sino a una fracción de ella. En el modelo de optimización se considera explícitamente la reasignación de usuarios, ya que al desactivar una BS se debe observar la afectación que esto traería para sus usuarios móviles asociados. La reasignación de usuarios introduce mayor complejidad al problema, ya que no solo el algoritmo propuesto debe decidir qué conjunto de BS se deben desactivar sino también que usuarios móviles se deben asociar a cada BS que permanece activa. Esto con el fin, de que con las BS que permanezcan activas se sigan ofreciendo el mayor número de servicios a los usuarios móviles activos. Al igual que el trabajo en [3], la reducción de energía se representa por el número de las BS activas. 
El artículo está organizado de la siguiente forma: la Sección 2 define el escenario de simulación y el modelo de optimización. La Sección 3 describe el algoritmo genético para la desactivación de las BS y reasignación de usuarios móviles. La Sección 4 muestra los experimentos realizados y los resultados obtenidos. Finalmente, la Sección 5 concluye el presente trabajo.

\section{Formulación del problema}

Un AG inicia con una población que es un conjunto de soluciones candidatas (individuos) al problema, las cuales se representan como una cadena (binaria, entera o flotante). A partir de algunos individuos de la población (padres) se crean nuevas soluciones (hijos). El objetivo de generar nuevos individuos es eventualmente encontrar mejores soluciones al problema. Cuando se aplica un AG se debe definir una función objetivo, que es una función matemática con la que se evalúa a cada solución candidata (individuo) para determinar qué tan apta es para resolver el problema. Otro elemento del AG son las restricciones que establecen si una solución es factible para el problema que se aborda [13].

En un AG que usa un modelo de población estacionario [13], se crean $n$ nuevos individuos, donde $n$ es menor al tamaño de la población. Dichos individuos son insertados a la población en el lugar de sus padres. En el AG de modelo de población estacionario que se usa en este trabajo, se crean dos hijos a partir de dos padres y se hace una comparativa de las aptitudes de los padres e hijos para que los dos individuos más aptos de los cuatro evaluados tomen el lugar de los padres [12].

En la Figura 1 se muestra el escenario de evaluación para este trabajo. Se observa que la red celular está compuesta por un número de estaciones base y de usuarios móviles desplegados sobre un área bidimensional con coordenadas cartesianas aleatorias que siguen una distribución uniforme para cada estación base $\left(B S_{j}\right)$ y cada usuario móvil $\left(U T_{i}\right)$. Para diferenciar la ubicación de estos dos componentes, una $B S_{j}$ usa la notación $\left(x_{j}, y_{j}\right)$, por otra parte, un $U T_{i}$ usa $\left(u_{i}, v_{i}\right)$. El total de estaciones base y usuarios móviles en la red en un instante de tiempo se denotan con la letra $J$ e $I$ respectivamente. Las BS pueden ser de dos tipos macro-celdas y femto-celdas. Los usuarios móviles asignados a una BS están delimitados dentro de su radio de cobertura $D$ (en la Figura 1, la BS y sus respectivos usuarios móviles son del mismo color). El radio de cobertura de una femto-celdas siempre será menor que el radio de cobertura de una macro-celda. Cuando una BS se apaga (ver $B S_{4}$ ) y alguno de sus usuarios móviles no se pudo reasignar a una nueva BS este se considera un usuario móvil sin servicio (ver $\left.U T_{10}\right)$.

La distancia euclidiana entre una $B S_{j}$ y un $U T_{i}$ se denota como $d_{j, i}$ y se calcula aplicando la ecuación (1):

$$
d_{j, i}=\sqrt{\left(u_{i}-x_{j}\right)^{2}+\left(v_{i}-y_{j}\right)^{2}}
$$




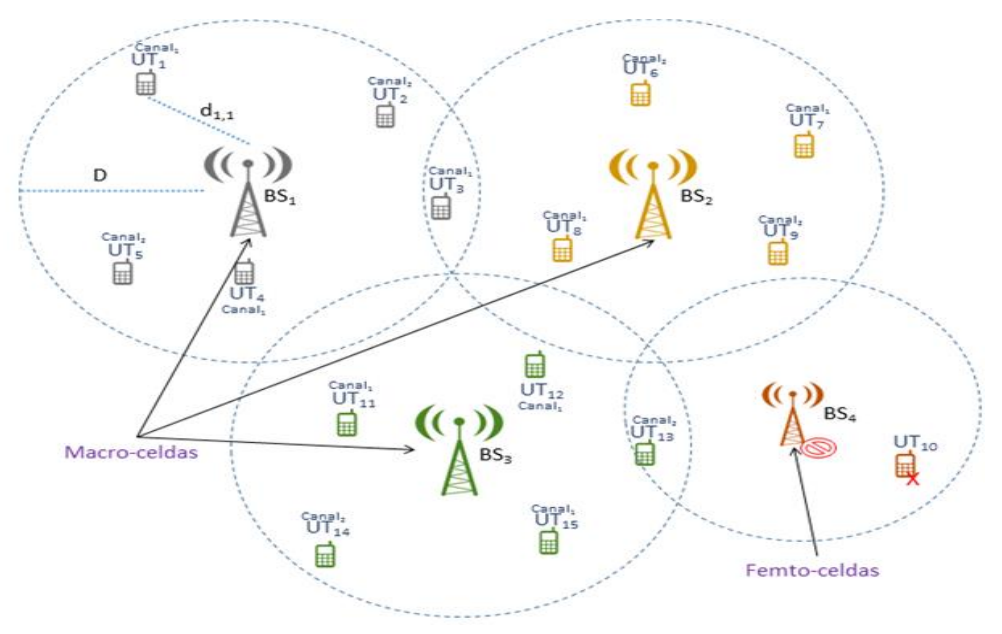

Fig. 1. Escenario de evaluación.

Cada $B S_{j}$ brinda servicio a varios $U T_{i}$ simultáneamente, para conocer esta relación se construyó la tabla $R B U$ (Relación Bases Usuarios) donde las filas representan a la $B S_{j}$ y las columnas a los $U T_{i}$. Si $R B U_{i, j}=1$, la $B S_{j}$ da servicio al usuario $U T_{i}$; en caso contrario, no hay relación entre el $U T_{i}$ y la $B S_{j}$. Tomando como referencia el escenario de la Figura 1, se construyó la Tabla $R B U$ mostrada en la Tabla 1. De esta forma, es posible observar cuando una $B S$ está apagada, como en el caso de la $B S_{4}$ (fila 4) pues solo hay ceros en sus casillas. También es posible establecer cuales $U T$ no están asociados a alguna $B S$, como es el caso del $U T_{10}$ el cual solo tiene valores iguales a cero en cada $B S_{j}$.

Tabla 1. $R B U$.

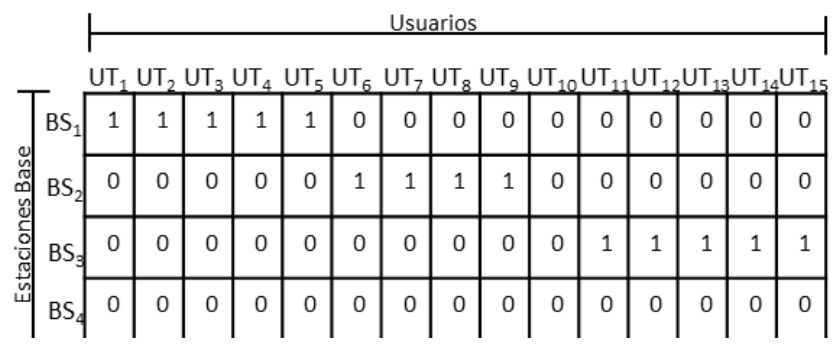

Una BS solo puede asignar una cantidad de canales $C$ y dar servicio a un determinado número de usuarios móviles. MUT es el número máximo de usuarios móviles a los que puede servir una BS. Las macro-celdas pueden servir a más usuarios móviles que las femto-celdas.

El vector binario $S B S$ representa a una solución candidata (individuo) del AG. Su longitud será igual al valor de $J$. La $B S_{j}$ está encendida si el elemento $S B S_{j}$ tiene un valor de 1 y apagada en caso contrario. En la Figura 2 se muestran algunos ejemplos de individuos y los escenarios a los que hacen referencia. Por otro lado, el vector $C U$ de 
longitud igual a $I$ contiene el identificador del canal que cada $U T_{i}$ tiene asignado por la BS que le da servicio. Los elementos en $C U$ podrán tomar un valor desde 1 hasta $C$.

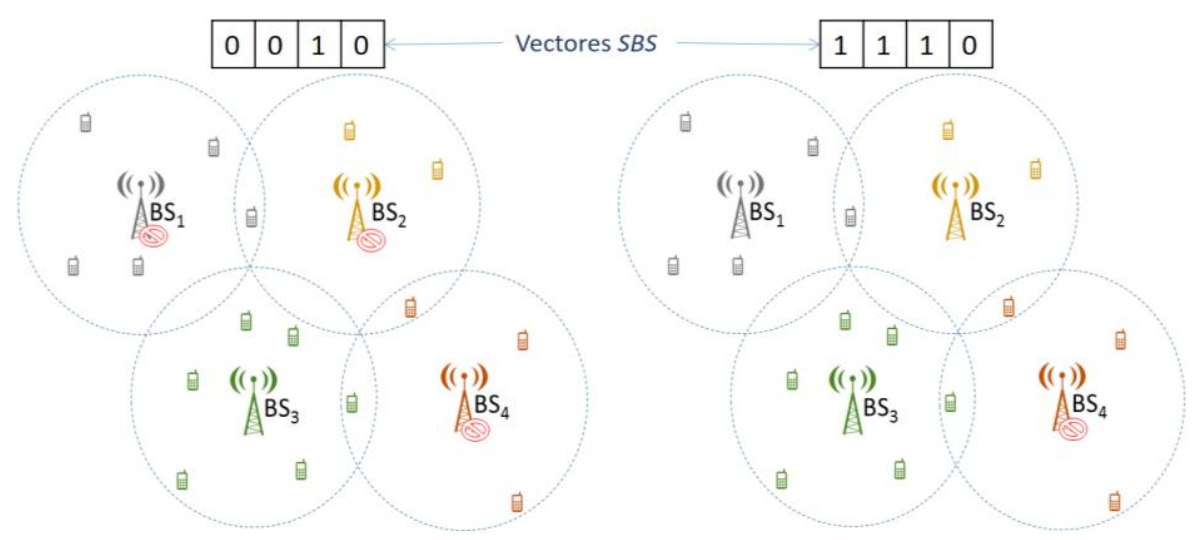

Fig. 2. Ejemplos del vector $S B S$ y sus escenarios correspondientes.

Con el fin de obtener el número mínimo de $B S$ activas que den servicio a la mayoría de los usuarios móviles en la red, se define la siguiente función objetivo:

$$
\text { Minimizar } \sum_{j=1}^{J} S B S_{j} \text {. }
$$

Como se muestra en (2), la solución es un vector $S B S$ que determine el menor número de BS encendidas. Por otro lado, para que una solución se considere factible al problema, debe de cumplir las siguientes restricciones:

$$
\begin{gathered}
\sum_{j=1}^{J} R B U_{j, i} \leq 1, \quad \forall i, \\
O n_{j}=1, \\
d_{j, i} \leq D, \forall i, \\
\sum_{i=1}^{I} R B U_{j, i} \leq M U T_{j}, \quad \forall j, \\
S N R_{j, i} \geq \alpha, \quad \forall i, \\
P I_{k} \leq(1-\beta) J, \quad \forall k .
\end{gathered}
$$

La restricción (3) limita a que un $U T_{i}$ sea servido por una sola $B S$. En la restricción (4) se establece que solo las BS encendidas podrán brindar servicio a los UT; esto es $O N_{j}=1$ si $\sum_{i=1}^{I} R B U>0$ y $S B S_{j}=1$, en caso contrario, $O N_{j}=0$. La distancia entre una $B S_{j}$ y un $U T_{i}$ al que se brinde servicio se limita en la restricción (5), donde esta no puede 
ser mayor al umbral de radio de cobertura $D$ de la BS en análisis. En (6) se obliga a una BS a no superar el máximo número de los usuarios móviles a los que puede dar servicio. La interferencia percibida por un $U T_{i}$ se representa con el valor de la relación señal a interferencia $(S N R)$ y debe ser mayor o igual a un umbral $\alpha$ como se establece en (7). Por último, el porcentaje de los usuarios móviles sin servicio al apagar algunas BS debe ser menor al umbral $\beta$ cumpliendo con (8), este porcentaje representa la probabilidad de interrupción $P I$.

\section{Algoritmo genético para desactivación de las BS y reasignación de usuarios móviles}

El procedimiento basado en un AG para desactivar las BS considerando la reasignación de usuarios móviles se muestra a continuación:

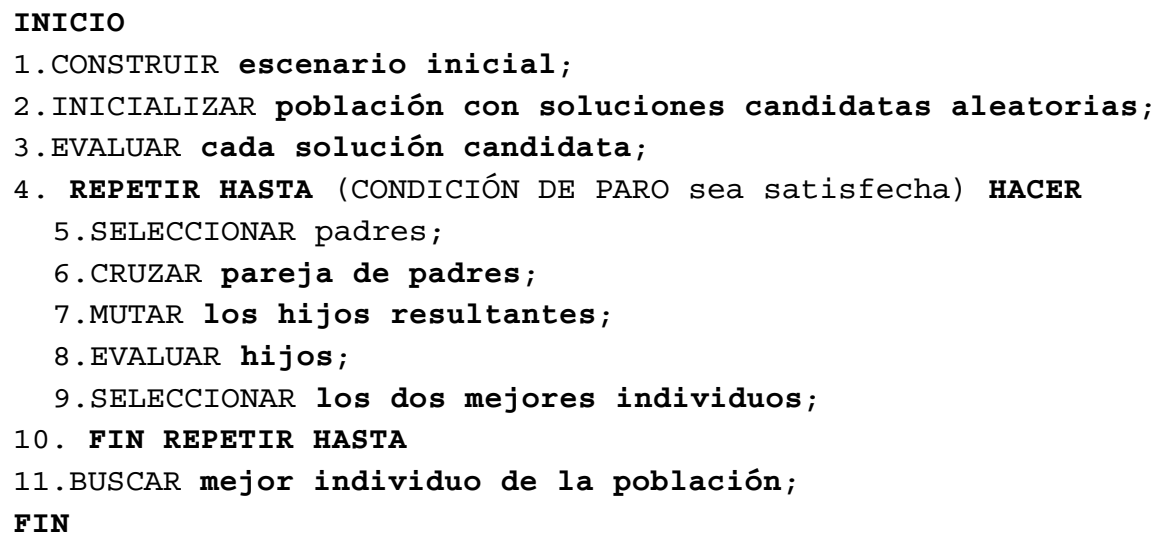

Para construir el escenario inicial (PASO 1), se despliegan aleatoriamente las $B S$ y los usuarios móviles dentro del área de cobertura. Enseguida, se especifica la cantidad de macro-celdas y femto-celdas que se incluirán la red. Se toma a cada uno de los usuarios móviles para ser asignados, un $U T_{i}$ es asignado a una $B S_{j}$ de acuerdo a: (1) si el total de los usuarios móviles a los que en ese momento da servicio la $B S_{j}$ es menor a $M U T$ y (2) si la distancia $d_{j, i}$ es menor o igual a $D$. Para elegir el canal asignado a cada $U T_{i}$ se genera un número aleatorio en el rango de $[1, C]$ y este valor se guarda en el vector $C U$ en la posición $i$.

El tamaño de la población se denota con NS. Se inicializa aleatoriamente al k-ésimo vector $S B S$ con valores binarios (PASO 2).

En el PASO 3 se evalúa al $k$-ésimo vector $S B S$ y su aptitud se representa por $A$. Con base al escenario inicial, se reasignan los usuarios móviles que estuvieron asociados a las BS apagadas en cada solución. Para ello, se realizan las siguientes acciones:

1. Identificar las $B S_{j}$ apagadas, es decir, los elementos del vector $S B S$ donde $S B S_{j}=0$.

2. Reasignar a los usuarios móviles asociados a una BS apagada a una BS activa, cumpliendo con las condiciones mostradas en las ecuaciones (3) - (7). 
3. Calcular $P I_{k}$ aplicando la ecuación (8). $P I_{k}$ es el porcentaje de los usuarios móviles que no fueron reasignados a una BS activa o aquellos usuarios móviles sin servicio en la solución $k$. Un $U T_{i}$ se considera sin servicio si todas las celdas de la columna $i$ en la tabla $R B U$ tienen un valor igual a 0 .

4. Evaluar (2), contando los elementos del vector $S B S$ donde $S B S_{j}=1$, es decir, las $B S_{j}$ activas.

Una solución puede contar con todas las BS encendidas o ninguna, sin embargo, soluciones con pocas BS encendidas o ninguna dejaría a muchos usuarios sin servicio, lo que se traduce en un mayor valor de $P I$. Por tanto, si $P I_{k}>\beta$, la solución $k$ será penalizada, es decir, su valor de $A_{k}$ se incrementará para que al comparar su aptitud con la aptitud de una solución factible esta quede en desventaja (las soluciones con menor aptitud son elegidas como padres). Una solución $k$ factible puede tener un valor máximo de $A_{k}=J$ (todas las $B S$ activas) por tanto a las soluciones no factibles o penalizadas se les sumará $(J+1)$ a su aptitud, con el fin de marcar una diferencia clara entre las soluciones factibles y no factibles. De esta manera se logra que el AG obtenga soluciones con pocas BS activas y que también cumplan con el umbral de $P I$ definido.

Cada solución $k$ cuenta con una tabla $R B U$ con la asignación de usuarios móviles a las $B S$ activas, los $U T_{i}$ sin servicio (si existen) y las $B S_{j}$ apagadas. Para calcular el valor de $S N R j$, al reasignar un $U T_{i}$ a una $B S_{j}$, se identifica el canal menos utilizado dentro de la $B S_{j .}$ y se aplica la ecuación (9):

$$
\begin{gathered}
S N R_{j, i}=P R_{j, i}-I T_{j, i}, \\
P R_{j, i}=P T-P L_{j, i}, \\
P L_{j, i}=A+B \log \left(d_{j, i}\right)+N,
\end{gathered}
$$

donde $P R_{j, i}$ es la potencia recibida en el $U T_{i} . I T_{j, i}$ es la interferencia total de los usuarios móviles que usan el mismo canal en la $B S_{j}$. En la ecuación (10), $P T$ es la potencia de transmisión de las BS. El valor de pérdidas por trayectoria $P L_{j, i}$ se determina a partir de (11) donde $A$ y $B$ son valores constantes, $N$ es un valor aleatorio distribución Gaussiana con una varianza de $\sigma_{2}$ que hace referencia al tipo de área donde se ubica la red (es mayor para una metrópolis y menor en espacios abiertos) [14]. En el presente trabajo se consideraron los siguientes valores $A=50, B=40$, y $\sigma_{2}=10$.

Seleccionar a los padres por la técnica de torneo [12] es el PASO 5 del algoritmo. El número de participantes en el torneo es de dos individuos.

Enseguida en el PASO 6 se genera un número decimal aleatorio en un rango de $[0,1]$ el cual es comparado con la probabilidad de cruzamiento $P C$. Si dicho número es menor o igual a $P C$ se procede a generar dos nuevos individuos con una combinación de los bits o elementos del vector $S B S$ de ambos padres. De manera más específica se utiliza el cruce por dos puntos [13].

Para el PASO 7 algunos bits de los hijos se mutan, un bit mutado toma su valor inverso ( 1 se convierte en 0 y 0 se convierte en 1 ). Para decidir que bits se mutaran, por cada elemento de los nuevos vectores $S B S$ se genera un número decimal aleatorio en 
un rango de [0,1]. Si este valor es menor a la probabilidad de mutación (PrM) el bit es cambiado.

Una vez que se han mutado los hijos, en el PASO 8 se procede a evaluar estas soluciones o vectores $S B S$. Esto es, se realiza la reasignación y cálculo del valor de $A_{k}$ para los dos nuevos individuos aplicando las cuatro acciones mencionadas en el PASO 3.

En el PASO 9 se compara el valor de $A_{k}$ de los dos padres y de los dos hijos. Los dos individuos con mejor valor de aptitud $A_{k}$ se insertan en la población en el lugar que ocupaban los padres.

En el PASO 10, se considera un ciclo al proceso de seleccionar a los padres, cruzarlos, mutar a los nuevos individuos y remplazar a los padres con los mejores individuos.

Existen distintas condiciones de paro, por ejemplo, si en el problema se conoce la solución óptima se puede forzar al algoritmo a realizar los ciclos que sean necesarios para encontrar dicha solución o una muy próxima a ella. En el caso de un AG con modelo de población estacionario, una condición de paro puede ser realizar los ciclos necesarios para que todos los individuos de la población se reemplacen por lo menos una vez por sus hijos. Debido a que no se conoce la solución óptima en el problema planteado y el cambiar a todos los individuos podría requerir de un número demasiado grande de ciclos, la condición de paro del algoritmo mencionada en el PASO 4 es realizar un determinado número de ciclos.

Por último, en el PASO 11 se busca en la población la solución con menor valor de $A k$. En caso de que existan dos o más soluciones con el menor número de $B S$ encendidas, se selecciona a la solución con menor probabilidad de interrupción $P I$.

\section{Resultados}

Se realizaron una serie de experimentos considerando los siguientes casos (i) una red homogénea (solo macro-celdas) y (ii) una red heterogénea (macro-celdas y femtoceldas). Este último caso se incluyó ya que como se ha reportado en [4], las femtoceldas sirven como soporte a las macro-celdas en estados de tráfico altos y se desactivan en caso contrario.

Tabla 2. Parámetros de simulación.

\begin{tabular}{|l|c|}
\hline Descripción & Valor \\
\hline Potencia de transmisión & $-40 \mathrm{~dB}$ \\
\hline Umbral de la relación señal interferencia $(\alpha)$ & $3 \mathrm{~dB}$ \\
\hline Área de cobertura & $25 \mathrm{~km} 2$ \\
\hline Radio de cobertura $(D)$ de las BS macro/femto & $2 \mathrm{~km} / 1 \mathrm{~km}$ \\
\hline Número de canales por BS & 8 canales \\
\hline Umbral de la probabilidad de interrupción $(\beta)$ & $1 \%$ \\
\hline Número máximo de usuarios móviles por macro-celda & 150 \\
\hline Número máximo de usuarios móviles por femto- celda & 75 \\
\hline
\end{tabular}


Se simula una red con $20 \mathrm{BS}$, divididas en 10 macro-celdas y 10 femto-celdas en redes heterogéneas. En todos los casos, se fue incrementando la cantidad de usuarios móviles activos en la red. Los parámetros de simulación considerados para la evaluación se muestran en la Tabla 2.

Tabla 3. Descripción de los experimentos.

\begin{tabular}{|c|c|c|c|}
\hline Experimento & $\begin{array}{c}\text { Número de } \\
\text { usuarios móviles }\end{array}$ & $\begin{array}{c}\text { Número de } \\
\text { macro-celdas }\end{array}$ & $\begin{array}{c}\text { Número de } \\
\text { femto-celdas }\end{array}$ \\
\hline \multicolumn{5}{|c|}{ Red Homogénea } \\
\hline 1 & 500 & 20 & - \\
\hline 2 & 200 & 20 & - \\
\hline 3 & 100 & 20 & 10 \\
\hline \multicolumn{5}{|c|}{ Red Heterogénea } \\
\hline 4 & 500 & 10 & 10 \\
\hline 5 & 200 & 10 & 10 \\
\hline 6 & 100 & 10 & $P=0$ \\
\hline
\end{tabular}

Para el AG se consideró una población de 50 individuos con una $P C=0.6$ y $\operatorname{PrM}=0.001$, además se realizan 2000 ciclos por cada ejecución del algoritmo. En la Tabla 3 se especifican las características de los 6 experimentos realizados. Cada experimento consta de 100 ejecuciones, en cada ejecución la posición de los usuarios móviles se modificó aleatoriamente y se obtuvo una solución $k$.

En los seis experimentos se observó que se reasignan los usuarios móviles de las BS apagadas a sus BS vecinas, y en la mayoría de las ejecuciones se mantiene con servicio al 99\% de los usuarios móviles presentes en la red. De igual forma el conjunto de las BS encendidas está distribuido en el área de cobertura de la red, es decir, las BS activas en las soluciones ofrecidas por el algoritmo no se encuentran en una sola región del área de cobertura o demasiado cerca una de otra. En el experimento 4 se mantuvieron encendidas BS muy cercanas solo cuando son heterogéneas, es decir, una femto-celda y una macro-celda.

En el experimento 1 se observó que, si una sola BS cubre una determinada área y no existen BS vecinas que puedan dar servicio a la mayoría de sus usuarios móviles, esta BS se mantiene encendida. Las BS encendidas no se seleccionan solo por el número de usuarios móviles a los que están sirviendo, sino por el número de usuarios móviles a los que puedan recibir de sus BS vecinas. Es decir, un BS se mantiene encendida no solo por tener muchos usuarios móviles relacionados en el escenario inicial, más bien se mantiene encendida porque al realizar la reasignación se logró incrementar considerablemente este valor.

En el experimento 4 se generó una mayor cantidad de tráfico en la red (mayor número de usuarios móviles). Se observa que una femto-celda da servicio a los usuarios móviles que se encuentran dentro del radio de cobertura de una macro-celda, es decir, una femto-celda da servicio a algunos usuarios móviles de la macro-celda.

En la Tabla 4, se reporta la solución con mejor aptitud obtenida durante las 100 ejecuciones de cada experimento. Así mismo, se reporta la peor solución obtenida y el promedio de las 100 ejecuciones. Para todos los experimentos realizados se observó 
que en promedio el número de BS activas se redujo considerablemente como se observa en la Tabla 4, de las 20 BS desplegadas inicialmente, solo se mantuvieron encendidas 10 BS o menos.

Tabla 4. BS encendidas para la mejor y peor corrida de cada experimento.

\begin{tabular}{|c|c|c|c|}
\hline Experimento & $\begin{array}{c}\text { BS encendidas/PI } \\
\text { (mejor ejecución) }\end{array}$ & $\begin{array}{c}\text { BS encendidas } \\
\text { (peor } \\
\text { ejecución) }\end{array}$ & $\begin{array}{c}\text { Promedio de } \\
\text { BS encendidas }\end{array}$ \\
\hline $\mathbf{1}$ & $6 \mathrm{BS} / 0.6 \%$ & $14 \mathrm{BS}$ & 9 \\
\hline $\mathbf{2}$ & $5 \mathrm{BS} / 1.0 \%$ & $11 \mathrm{BS}$ & 7 \\
\hline $\mathbf{3}$ & $5 \mathrm{BS} / 0.0 \%$ & $11 \mathrm{BS}$ & 7 \\
\hline $\mathbf{4}$ & $8 \mathrm{BS} / 0.2 \%$ & $15 \mathrm{BS}$ & 10 \\
\hline $\mathbf{5}$ & $6 \mathrm{BS} / 0.5 \%$ & $13 \mathrm{BS}$ & 8 \\
\hline $\mathbf{6}$ & $5 \mathrm{BS} / 1.0 \%$ & $14 \mathrm{BS}$ & 8 \\
\hline
\end{tabular}

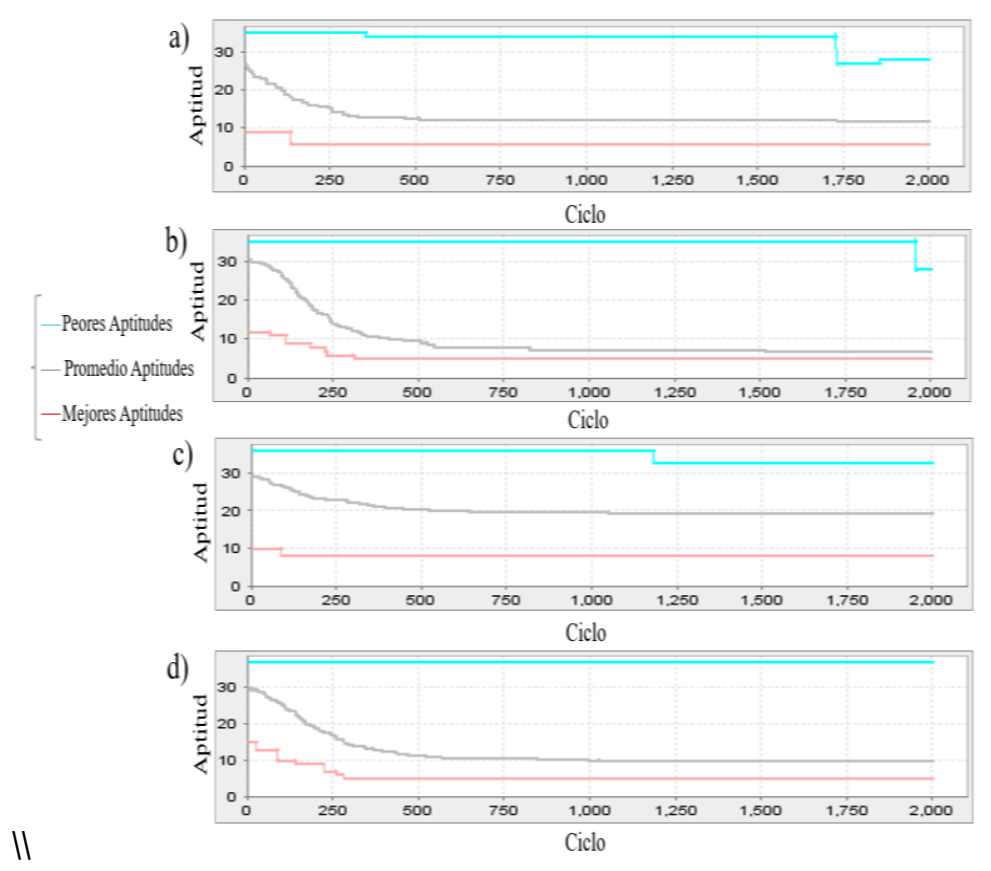

Fig. 3. Convergencia del algoritmo: a) experimento 1, b) experimento 3, c) experimento 4, d) experimento 6 .

Al disminuir el número de usuarios móviles también se reduce el número de BS activas para ambos tipos redes. Por ejemplo, en redes homogéneas al reducir de 500 a 200 usuarios móviles, el número de BS encendidas disminuye de 6 a 5 . En el caso de redes heterogéneas se reduce de 8 a 5 BS encendidas (ver Tabla 4). En el experimento 5 se logran apagar más BS en la mejor corrida que en el experimento 2, puesto que la 
mayoría de las BS apagadas eran femto-celdas. Entonces el algoritmo decide apagar una BS con menores capacidades (radio de cobertura y MUT).

Con respecto al número de usuarios móviles reasignados no se observa diferencias significativas al comparar los dos tipos de redes. Sin embargo, cuando hay 100 usuarios móviles en la red, en promedio se resignan a 36 de ellos; por otra parte 75 de los 200 usuarios móviles presentes en los experimentos 2 y 5 fueron reasignados; y para los experimentos con 500 usuarios móviles en promedio se reasignaron 165 usuarios móviles. Entonces al apagar las BS se reasigna a casi el 50\% de los usuarios móviles presentes en la red.

En el experimento 4, de las 12 BS apagadas, 8 eran femto-celdas, mientras que para el experimento 5 todas las femto-celdas fueron apagadas. Entonces para redes heterogéneas cuando el número de usuarios móviles disminuye, las BS apagadas son femto-celdas.

En el $100 \%$ de las ejecuciones del algoritmo en redes homogéneas se obtienen soluciones donde se cumple con $P I \leq 1 \%$, en cambio, en redes heterogéneas solo en un $70-90 \%$ de las ejecuciones se obtiene soluciones que cumplen dicha relación. Entonces es más complejo realizar el apagado de BS en redes heterogéneas.

En la Figura 3 se muestra la convergencia de la mejor solución encontrada en los experimentos 1, 3, 4 y 6 . Además se muestra el promedio y la peor aptitud por ciclo. Se observa que conforme aumenta el número de usuarios móviles es más difícil para el algoritmo evolucionar o converger. Esto se debe a que el número de soluciones válidas disminuye dado que las soluciones están estrechamente ligadas al número de usuarios móviles presentes en el área.

\section{Conclusiones y trabajo futuro}

En el presente trabajo se abordó el problema de desactivación de BS implementando un algoritmo genético con modelo de población estacionario. Esto con el objetivo de minimizar el número de BS encendidas considerando la reasignación de usuarios móviles, para redes homogéneas y heterogéneas.

Conforme se incrementa la cantidad de usuarios móviles en la red, es más complejo encontrar una solución. Lo anterior se debe al proceso de reasignación de usuarios móviles incluido en este trabajo. Por este motivo, es justificable utilizar la técnica de AG con población estacionaria, ya que en cada ciclo de evaluación solo considera a dos padres y a dos hijos, a diferencia de un AG con población generacional.

Los experimentos realizados con diversa densidad de tráfico (número de usuarios móviles activos) y distintos tipos de red muestran que:

- Se puede apagar hasta un 50\% del número de BS encendidas cuando los estados de tráfico son bajos tanto en redes homogéneas como heterogéneas.

- Después de apagar las BS se reasignaron entre 33-38\% de los usuarios móviles presentes en la red, por este motivo la reasignación de usuarios móviles debe ser considerado un factor de impacto en soluciones que involucren desactivación de BS en redes celulares. 
- Es posible dar servicio a casi el 100\% de los usuarios móviles aun si se apagan ciertas BS de una red.

- En redes heterogéneas el algoritmo decide desactivar primero a las BS femto-celdas cuando el tráfico es menor, lo que coincide con lo reportado en la literatura, en la que se menciona que su uso es más beneficioso cuando la red macro-celular presenta estados de tráfico alto.

Como trabajo futuro se plantea un análisis de sensibilidad con el umbral de la relación señal-interferencia para estimar hasta qué punto se puede garantizar QoS para los usuarios móviles.

\section{Referencias}

1. Cisco VNI Complete Forecast. Disponible en: http://www.cisco.com/c/dam/assets/ $\mathrm{sol} /$ service-provider/vni-complete-forecast/vnisp.html

2. El-Beaino, W., Al-Kanj, L., El-Hajj, A. M., Dawy, Z.: Optimized joint cell planning and BS on/off switching for LTE networks. 12(16), pp. 1537-1555 (2015)

3. Azzam, S. M., Elshabrawy, T.: Re-Dimensioning Number of Active eNodeBs for Green LTE Networks Using Genetic Algorithms. In: Proceedings of European Wireless 2015, 21th European Wireless Conference, pp. 1-6 (2015)

4. Alsharif, M. H., Nordin, R., Ismail, M.: Survey of Green Radio Communications Networks: Techniques and Recent Advances. J. Comput. Netw. Commun. 2013, e453893, doi:10.1155/2013/453893 (2013)

5. Akram, B. S., Furkan, A., Halim, Y.: A genetic algorithm based cell switch-off scheme for energy saving in dense cell deployments. IEEE Globecom Workshops (2012)

6. James, J. Q., Yu, V. O. K. L.: Base Station Switching Problem for Green Cellular Networks with Social Spider Algorithm. In: IEEE Congress on Evolutionary Computation (CEC), Beijing (2014)

7. Pampara, G.: Angle modulated population based algorithms to solve binary problems. Disponible en: http://repository.up.ac.za/handle/2263/22801 (2012)

8. Kyuho, S., Hongseok, K., Bhaskar, K., Yung, Y.: Base Station Operation and User Association Mechanisms for Energy-Delay Tradeoffs in Green Cellular Networks. 29, 1525-1536 (2011)

9. Seonwook, K., Sunghyun, C., Byeong, G. L.: A Joint Algorithm for Base Station Operation and User Association in Heterogeneous Networks. IEEE Communications Letters, 17, pp. 1552-1555 (2013)

10. Talbi, E. G.: Metaheuristics: From Design to Implementation. John Wiley \& Sons (2009)

11. Oh, E., Son, K., Krishnamachari, B.: Dynamic Base Station Switching-On/Off Strategies for Green Cellular Networks. IEEE Trans. Wirel. Commun. 12, pp. 2126-2136 (2013)

12. Neal-Holtschulte, M. M.: Should every man be an island? In: GECCO, Amsterdam, The Netherlands (2013)

13. Eiben, A. E., Smith, J. E.: Introduction to Evolutionary Computing. Springer Berlin Heidelberg (2015)

14. Stephen, B., Wicker, B. K.: Experimental analysis of local search algorithms for optimal base station local (2000) 